ceria crystals are anchored to the BHA and are separated from one another, even at very high temperatures they cannot fuse together. The ceria-coated BHA has a light-off of just about $400^{\circ} \mathrm{C}$ and can withstand temperatures higher than $1100^{\circ} \mathrm{C}$. The catalyst is stable in the presence of water vapor and other potential poisons.

"This combination of low-temperature catalytic activity, high-temperature thermal stability, and poisoning resistance renders our catalysts interesting for potential practical applications in ultralean catalytic combustion of methane," the authors conclude. Ying said that the procedure for creating the material paves the way for materials that could improve other high-temperature processes such as the production of some chemicals.

\section{Positrons Help Locate Nano-Surface Defects in Gold}

In a paper published in the November 29 issue of Physical Review Letters, a team from Oak Ridge National Laboratory, Lucent Technologies, Fisk University, and Japan's Electrotechnical Laboratory document an experiment using positrons to find clusters of four atomic vacancies at the surface of gold nanoparticles embedded in a magnesia matrix. These clusters of vacancies explain changes in the optical properties when the materials are subjected to different fabrication processes.

Positrons were generated by smashing gamma rays against a tungsten target. The gamma rays divide into negatively charged electrons and their antimatter, positrons. The decay of unstable sodium22 provided an alternative source of positrons. The positrons are injected into the gold nanoparticles, and through advanced spectroscopy, the researchers are able to determine the size, location, and concentration of the vacancy clusters. According to the researchers, possible future applications for this work include higher-speed computer chips than available now, manipulation of the properties of optical devices, less brittle ceramic material than currently available, and improved fiber-composite materials than currently available.

\section{Spray-on Skin of Polymer Fibers May Allow Wounds to Heal Without Scarring}

Researchers at Electrosols, a biotechnology company based in Haslemere, Surrey, United Kingdom, have developed a spray that could help wounds heal without scarring. The spray produces a fine web of biodegradable polymer fibers that collagen-making cells called fibroblasts can grow on. As more and more fibroblasts grow on the polymer webbing, they produce a regular collagen structure, much like that in normal skin. Electrosols researcher Ron Coffee believes that controlling the formation of collagen in this way will lead to normal skin growth instead of scarring.

As reported in the January 8 issue of New Scientist, to make the spray, Coffee mixes ethanol and a biodegradable polymer-such as polylactic acid-in a small semiconducting container, and then gives it an electric charge by putting an electric field across the container. Because the wound is at a far lower electrical potential than the polymer, the solution is attracted to the skin surface and flies out through tiny nozzles, producing fine, light fibers, each of them $5 \mu \mathrm{m}$ in diameter. The fibers have the same charge, so they repel each other, making them regularly spaced.

Other researchers are more cautious about the spray's prospects. Bruce Martin, a reconstructive surgeon at the University of Florida, said, "This initial polymer fiber mat wouldn't necessarily have any bearing on the final scar. Collagen is organized and reorganized continuously, and that's governed by a whole range of things."

When skin is punctured, the damage often destroys the weave-like structure of collagen that gives skin its strength. But when the body tries to patch up the wound the body creates a quick fix by producing thin, aligned strips of collagen. When skin cells grow on this, they produce the pale, less flexible material known as scar tissue, rather than normal skin.

\section{Uniformity of Rocks at Lower Levels of Deep Boreholes May Facilitate Burial of Radioactive Waste}

Fergus Gibb, a geologist at the University of Sheffield, United Kingdom, proposes that high level radioactive waste (HLW) should be disposed of in boreholes over $4 \mathrm{~km}$ deep. As reported in the January issue of the Journal of the Geological Society, special cylinders filled with HLW are placed in the lower section of the hole, which is then back-filled with crushed granite and sealed. The container's contents are designed to deliver the heat necessary to heat the waste and surrounding rock such that maximum temperatures of $800-900^{\circ} \mathrm{C}$ are generated at the container/rock interface. At these temperatures, the rock is changed in a series of fronts moving away from the container, followed, in the zone closest to the container, by partial melting. As the heat decays away, this partial melt solidifies, sealing the container and its con-

\section{S $\quad T$ A I INSTRUMENTS}
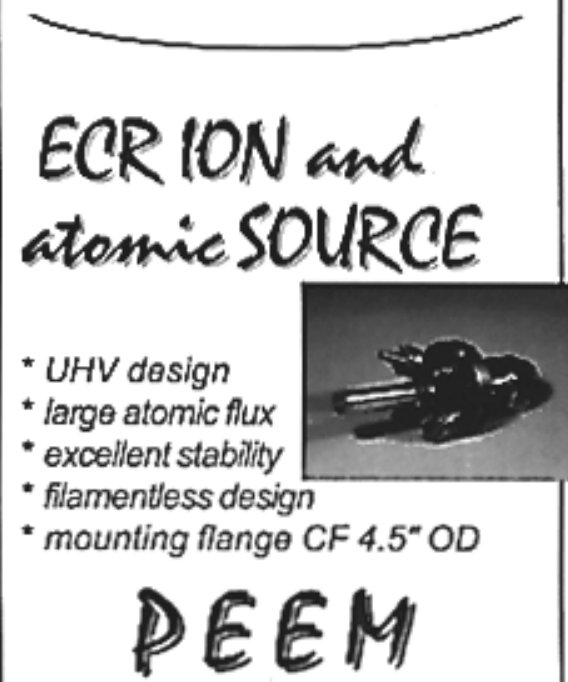

Photo Emission Electron Microscope

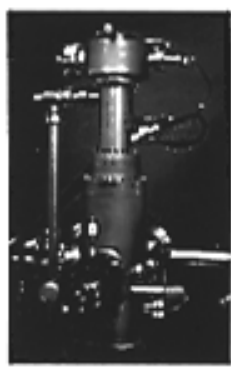

* modular design

* energy filtering

* photoelectron spectroscopie

- chemical imaging

* high spatial resolution
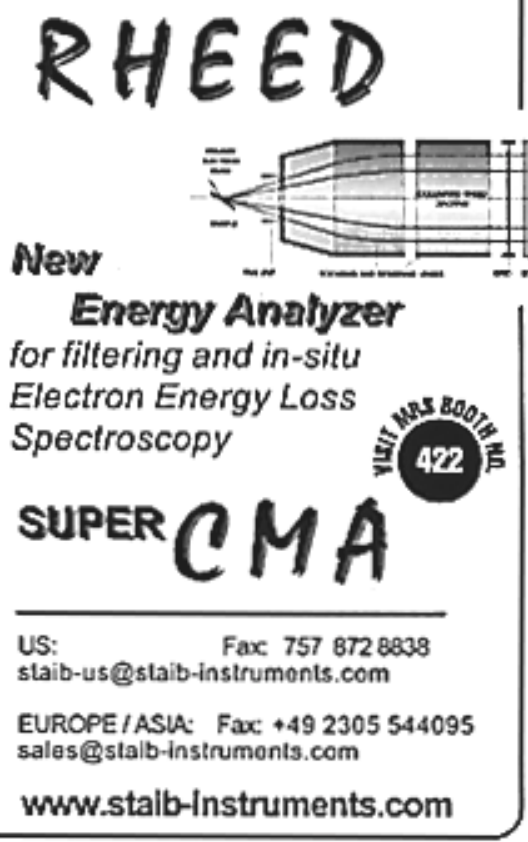

CIrcle No. 13 on Inside Back Cover 